\title{
Chromatographie en couches minces des 2,4-dinitrophénylhydrazones des composés carbonylés
}

\author{
par \\ G. PRADEL et J. ADDA \\ I.N.R.A. \\ Laboratoire de Recherches Fromagères, Chemin de Saint-Etienne \\ 15000 Aurillac \\ Laboratoire d'Etude des Arômes, C.N.R.Z. - 78350 Jouy-en-Josas
}

Le rôle des composés carbonylés dans l'acquisition des qualités organoleptiques ou dans l'apparition des défauts d'arôme des produits alimentaires est unanimement reconnu et de nombreux travaux y ont été consacrés.

Leur présence dans les produits laitiers a été signalée dans tous les isolats d'arôme et le rôle des méthylcétones a été plus particulièrement souligné dans les pâtes persillées : Anderson (1966), Anderson et Day (1966), Dartey et Kinsella (1971), Morgan et Anderson (1956), Patton (1950), Sato et al. (1966), Schwartz et Parks (1963), Svensen et Ottestad (1969).

Les composés carbonylés peuvent être étudiés sous forme libre mais leur séparation et leur identification se sont avérées souvent plus faciles par l'intermédiaire de dérivés caractéristiques (formés par action du sulfite acide, de l'hydroxylamine, de l'hydrazine et ses dérivés, du réactif $\mathrm{T}$ de Girard et Sandulesco) qui permettent, si besoin est, de régénérer le composé initial.

Parmi tous ces dérivés caractéristiques, les 2,4-DNPHones ont été et de loin les plus utilisés. Pour leur formation qui a lieu en milieu acide, différents protocoles ont été établis : Forss et al. (1955), Haverkamp Begemann et de Jong (1959), Huelin (1952). Le choix d'une solution aqueuse ou d'un solvant comme milieu réactionnel dépend des conditions dans lesquelles les composés carbonylés ont été isolés et de la nature des opérations de séparation et d'identification qu'ils subiront par la suite. En présence d'un excès de 2,4-DNPHine,

\footnotetext{
Abréviations : 2,4-DNPHone $=$ 2,4-dinitrophénylhydrazone.

2,4-DNPHine $=2,4$-dinitrophénylhydrazine.

P.E.G. = polyéthylène glycol.
} 
les composés dicarbonylés donnent un dérivé bis-2,4-DNPHone (ou osazone). Toutefois dans des conditions bien définies que Reich et Hefle (1956) ont contribué à préciser, il est possible d'obtenir le dérivé mono-2,4-DNPHone, dont seule une fonction carbonyle a réagi avec la 2,4-DNPHine.

Les 2,4-DNPHones offrent l'avantage de transformer de façon quantitative les composés carbonylés, pour la plupart très volatils, en composés cristallisant à la température ambiante et de plus insolubles en milieu aqueux. Ces dérivés, de par leurs propriétés physico-chimiques et notamment du fait de leur coloration naturelle ressortissent plus particulièrement aux techniques de chromatographie en phase liquide bien qu'il soit également possible de les séparer par chromatographie en phase gazeuse : Kallio et al. (1972), Papa et Turner (1972), Soukup et al. (1964).

La chromatographie en couche mince qui peut être mise en œuvre avec des quantités de substance relativement faibles a été très largement utilisée. En appliquant cette technique, les divers auteurs ont utilisé des systèmes chromatographiques très variés adaptés à la séparation de la catégorie de composés carbonylés dominante dans le produit étudié.

Nous voudrions d'abord souligner qu'au travers de leur diversité, les systèmes proposés se fondent en réalité sur une unité de principes et qu'en dépit de cette diversité, seuls quelques systèmes sont réellement utilisés en pratique.

Notre propos sera ensuite de montrer qu'il existe des compatibilités entre des systèmes conçus indépendamment et qu'il est possible de réaliser des enchaînements chromatographiques permettant de séparer une gamme étendue de composés carbonylés. Ces composés pourront différer entre eux par la structure de leur chaîne carbonée, par le nombre et la position des fonctions carbonyles, par leur degré d'insaturation et aussi par l'existence, chez certains, de substituants hydroxyle.

Qu'ils aient été clairement précisés par les auteurs ou qu'ils aient été admis implicitement, un certain nombre de principes chromatographiques sont sous-jacents à ces travaux :

- Les propriétés physico-chimiques des substances déterminent leur comportement chromatographique ; à la définition de classes fondées sur des propriétés chimiques correspond sur le plan chromatographique la mise en évidence de comportements propres à chaque classe.

- Parmi les différents types de liaison que les substances peuvent établir avec le support chromatographique, ce sont celles résultant de l'adsorption qui peuvent le mieux être responsables du comportement caractéristique d'une classe donnée. 
- Dans un composé carbonylé, plus la chaîne carbonée sera longue, plus le caractère polaire conféré à la molécule par la fonction carbonyle et éventuellement par la présence de doubles liaisons, ou de fonctions hydroxyle, s'en trouvera atténué. En d'autres termes, à l'intérieur d'une classe, le caractère apolaire (ou lipophile) ira croissant des homologues inférieurs aux homologues supérieurs : il variera avec leur poids moléculaire. Le partage de substances organiques entre deux solvants de polarité différente sera fonction de la polarité de ces substances. La chromatographie de partage qui se fonde sur les variations du coefficient de partage selon la polarité des substances semble être la technique la mieux adaptée à la séparation d'homologues qui d'un point de vue chromatographique diffèrent essentiellement par leur polarité.

C'est en combinant chromatographies d'adsorption et chromatographies de partage que les auteurs ont obtenu la séparation de mélanges plus ou moins complexes.

\section{SEPARATION D'UN MELANGE COMPLEXE}

Le schéma de séparation ci-dessus énoncé, simple dans son principe parce que calqué sur la classification chimique des composés se complique dans la pratique du fait du grand nombre de classes susceptibles d'être représentées et de la gamme étendue d'homologues que peut compter chaque classe.

Dans une séparation de classes comportant des homologues de poids moléculaire très différents, les homologues à courte chaîne migrent moins que les homologues à longue chaîne et peuvent dans les cas extrêmes se retrouver dans la classe à $R_{F}$ immédiatement inférieur au niveau des homologues supérieurs de cette classe. Ainsi, dans un système ou alkanones (cétones saturées), alkanals (aldéhydes saturées) et alk-2-énal s'étagent dans l'ordre des $R_{F}$ décroissants, la 2-propanone (acétone) et la 2-butanone se mêleront aux homologues à longue chaîne des aldéhydes saturées tandis que le méthanal (formaldéhyde) et l'éthanal (acétaldéhyde) resteront au niveau des homologues supérieurs des alk-2-énals. Les bandes représentatives des classes ne seront plus alors nettement séparées.

Le fractionnement en classes ne pourra être obtenu que si à l'intérieur de chaque classe, les homologues ont un nombre voisin d'atomes de carbone, compris dans une fourchette caractéristique du système utilisé.

Dans une situation de complexité maximale, c'est-à-dire celle où plusieurs classes de composés aliphatiques sont représentées, la séparation (fig. 1) comportera dans ses grandes lignes :

(A) Le fractionnement en groupes de classes aboutissant à l'isolement des aliphatiques. 
A. Groupes de classes

B. Sous-groupes de classes

C. Classes

D. Homologues

\section{MELANGE COMPLEXE}

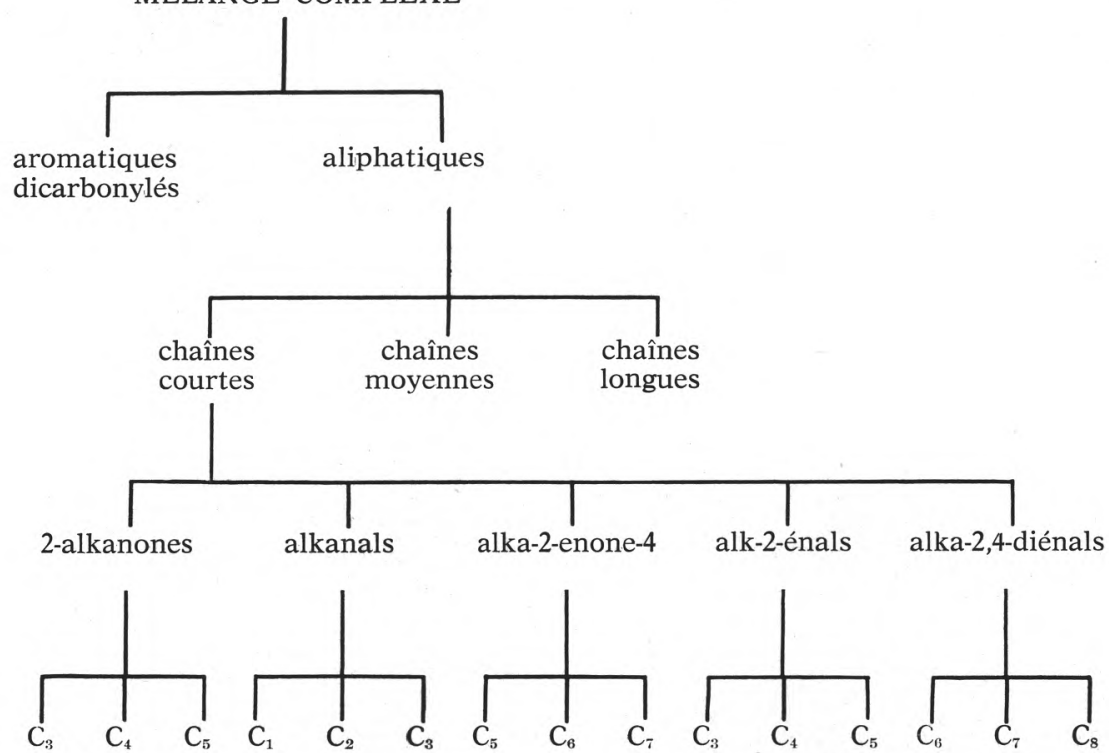


(B) Une seconde étape au cours de laquelle le groupe des classes d'aliphatiques précédemment isolé sera fractionné en plusieurs sousgroupes constitués par des composés - appartenant à des classes différentes - mais ayant des longueurs de chaîne voisines. Généralement il sera ainsi déterminé trois sous-groupes : les composés à chaînes courtes, les composés à chaînes moyennes et les composés à chaînes longues.

(C) La séparation des sous-groupes de classes en classes.

(D) La séparation des classes en leurs homologues.

En ce qui concerne les composés aromatiques et dicarbonylés, le nombre limité d'homologues d'une classe donnée, voire l'absence te classe, autorise des simplifications à ce protocole qui se traduisent par la suppression de l'étape (B) dans la séparation des dicarbonylés et des étapes (B) et (C) dans la séparation des aromatiques.

\section{I. - Fractionnement en groupes de classes (fig. 2)}

Dhont et Dijkman (1967) étudiant les composés carbonylés aromatiques ont proposé pour l'étape de fractionnement en groupes de classes, une succession de deux systèmes chromatographiques scindant le mélange complexe en deux fractions : l'une contenant les 2,4-DNPHones des composés carbonylés aliphatiques (fraction 1), l'autre les 2,4-DNPHones des composés carbonylés aromatiques et des composés dicarbonylés (fraction 2).

Le premier système (système I) utilise les propriétés d'adsorption d'un mélange en proportions égales d'oxyde de magnésium et de Kieselguhr-G (solvant hexane-chloroforme 7:3). Dans ce système les bandes des composés aliphatiques ont un $\mathrm{R}_{\mathrm{F}}$ supérieur à celui de la p-tolualdéhyde utilisée comme marqueur tandis que les composés dicarbonylés sous forme de dérivés bis ainsi que la plus grande partie des composés carbonylés aromatiques ont un $\mathrm{R}_{\mathrm{F}}$ inférieur à celui du composé marqueur.

$\mathrm{Au}$ cours de nos essais nous avons noté que les composés carbonylés hydroxylés, tels la 3-hydroxy 2-butanone (acétoïne) étaient fortement adsorbés et se retrouvaient dans la fraction des aromatiques (fraction 2).

Dans la fraction 1, en plus des aliphatiques sont encore présents les composés carbonylés aromatiques dont la fonction carbonyle est située sur une chaîne latérale : 2-phénylpropanal et acétophénone par exemple. Aussi cette fraction fera-t-elle à nouveau l'objet d'une séparation par chromatographie de partage sur Kieselguhr G imprégné de 2-phénoxyéthanol (solvant éther de pétrole $60^{\circ}-80^{\circ}$ ). Dans ce système (système II) décrit pour la première fois par Urbach (1963), les composés carbonylés aromatiques non isolés précédemment de même que le dérivé mono du diacétyle qui ont un $\mathrm{R}_{F}$ inférieur à celui de l'acétaldéhyde utilisée comme marqueur se trouvent séparés 


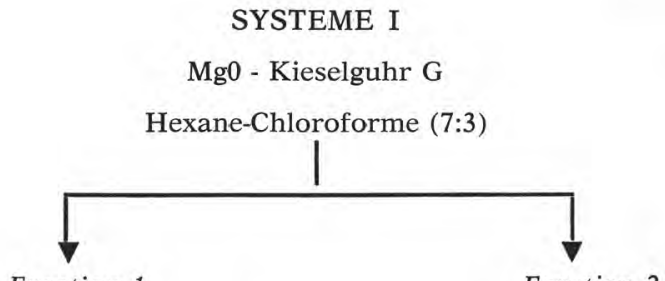

Fraction 1

Fraction 2

migrent plus que la p-tolualdéhyde

- aliphatiques

- aromatiques à chaîne latérale

- dicarbonylés (dérivés mono)

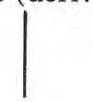

Système II

2-phénoxyéthanol-Kieselguhr G

Ether de pétrole $60^{\circ}-80^{\circ}$

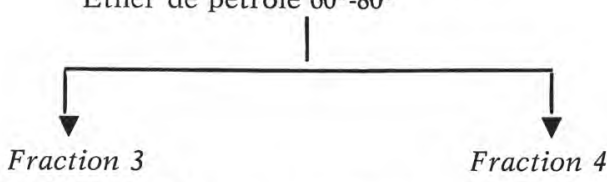

migrent plus que l'acétaldéhyde

- aliphatiques

- dicarbonylés (dérivés mono)

migrent moins que l'acétaldéhyde

- aromatiques à chaîne latérale

- diacétyle (dérivé mono)

- terpéniques

migrent moins que la p-tolualdéhyde

- aliphatiques hydroxylés

- aromatiques

- dicarbonylés (dérivés bis)

- alk-2-énals (les premiers termes)

- méthanal

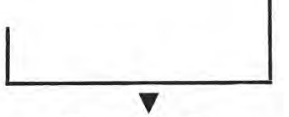

Fraction 5

fig. 2

Fractionnement en groupes de classes des composés carbonylés

de la fraction 3 et constituent la fraction 4. Le méthanal, du fait de son caractère très polaire, reste dans cette dernière fraction et il $\mathrm{y}$ a toutes raisons de penser que les alk-2-énals tels le 2-propenal (acroléine) et le 2-butenal (crotonaldéhyde) accompagnent également cette fraction.

Après avoir réuni les fractions 2 et 4 en une seule fraction 5 , le mélange initial se trouve séparé en :

- Une fraction 5 caractérisée par la présence des composés carbonylés aromatiques et des composés dicarbonylés sous forme de dérivés bis et pouvant contenir en outre :

des acides cétoniques,

le dérivé mono du diacétyle,

les composés carbonylés aliphatiques hydroxylés,

les alkanals et les alk-2-énals les plus polaires. 
- Une fraction 3 caractérisée par la présence des composés carbonylés aliphatiques et dans laquelle peuvent être présents :

des composés carbonylés terpéniques,

des dérivés mono des composés dicarbonylés.

En fait le nombre de classes représentées peut se trouver réduit. C'est ainsi qu'en raison de leur individualité propre (propriétés physico-chimiques et rôle biologique originaux) les acides cétoniques ne sont pas considérés comme des composés carbonylés types. Ils pourront donc être éliminés de la préparation initiale.

Par ailleurs, les conditions généralement retenues pour la formation des 2,4-DNPHones, notamment l'excès de 2,4-DNPHine ne favorisent pas l'apparition de dérivés mono.

Enfin le nombre de classes représentées pourra également se trouver réduit par suite de l'origine de l'échantillon : ainsi dans le cas de produits laitiers qui nous intéresse les composés carbonylés terpéniques ne seront pas représentés.

Dans une application que nous avons faite de ces techniques, l'absence conjuguée de composés carbonylés aromatiques à chaîne latérale, de dérivés mono des composés dicarbonylés, de composés carbonylés terpéniques et de composés carbonylés aliphatiques hydroxylés nous a même permis de faire l'économie du système II. Nous obtenions ainsi une séparation complète entre aliphatiques, d'une part (fraction 1) et aromatiques et dicarbonylés d'autre part (fraction 2).

Le système II altère la séparation des aliphatiques; les premiers termes des alka-2-enals et le méthanal sont entraînés avec les aromatiques et les dicarbonylés, l'intervention de ce système ne peut donc être justifiée que par la recherche d'une meilleure séparation des aromatiques présents.

Certains auteurs, notamment Day (1965) ont préconisé pour séparer les composés aliphatiques des composés dicarbonylés (et implicitement des composés aromatiques qui accompagnent ces derniers) d'extraire d'abord par l'hexane le mélange initial des 2,4DNPHones en solution aqueuse. Seuls les composés aliphatiques sont solubles dans l'hexane. Une seconde extraction du mélange initial par le benzène ou le chloroforme isole les composés dicarbonylés et aromatiques. Au cours de nos essais, nous avons soumis des mélanges de composés carbonylés à cette double extraction et pour chacun des extraits hexane et benzène nous avons réalisé le fractionnement en groupes de classes à l'aide des systèmes I et II (fig. 2), décrits ci-dessus. Les résultats ont fait apparaître que si la fraction hexane ne contenait que des composés carbonylés aliphatiques, elle ne les contenait pas tous. Après plus de 25 extractions successives nous avons noté qu'il subsistait encore des quantités appréciables de composés carbonylés aliphatiques dans le mélange initial. Ces compo- 
sés étaient extraits au cours de la phase suivante en compagnie des dicarbonylés et des aromatiques. De plus le spectre des aliphatiques de la phase benzénique différait de celui des aliphatiques de la phase hexanique. L'utilisation d'extraction "sélective " pour la séparation en groupes classes ne nous paraît donc pas offrir une véritable alternative à la chromatographie en couches minces.

\section{II. - Séparation des composés monocarbonylés aliphatiques}

La séparation des composés carbonylés aliphatiques est entreprise dans le cas le plus général sur la fraction 3 (fig. 2). Néanmoins lorsque se présentent les simplifications dont il a été fait état ci-dessus, elle devient possible dès la fraction 1, sans que soit pour autant compromise la séparation des autres composés.

Historiquement, c'est d'abord par chromatographie sur papier et par chromatographie sur colonne que les auteurs ont mené à bien la séparation des composés carbonylés aliphatiques. Aussi les premières réalisations en couches minces ont-elles consisté en une transposition directe de ces deux techniques, avec utilisation du même adsorbant ou de la même phase stationnaire.

\section{1) Fractionnement des composés monocarbonylés aliphatiques en classes}

Adaptant à la couche mince les techniques de Forss et al. (1960) qui fractionnaient en classes des composés carbonylés aliphatiques sur colonne d'alumine, Urbach (1963) séparait par chromatographie d'adsorption sur couche mince d'oxyde d'aluminium, les aldéhydes, les 2-alkanones (méthylcétones saturées) et les alk-1-en-3-ones (vinylcétones) avec comme solvant un mélange éther de pétrole $\left(30^{\circ}-40^{\circ}\right)$ oxyde diéthylique $(96: 4)$. Ce système présentait une insuffisance notable: il ne fractionnait pas les différentes classes d'aldéhydes saturées et insaturées. Pour y remédier, l'auteur, s'inspirant des techniques mises au point par Barret et al. (1962) et De Vries (1962) proposait une chromatographie sur couche mince d'alumine imprégnée de nitrate d'argent qui reprenait le groupe des aldéhydes, le scindait en alkanals, alk-2-énals, alka-2,4-diénals et certains alka-2,6diénals.

Ces derniers auteurs dans leurs travaux sur les lipides étaient en effet parvenus à séparer, chez les glycérides insaturés, des isomères géométriques et des isomères de position, par chromatographie en couche mince sur gel de silice imprégné de nitrate d'argent. Ce faisant, ils réalisaient avant la lettre une chromatographie d'affinité fondée sur la propriété des doubles liaisons de former avec le nitrate d'argent des composés de coordination (complexes).

S'appuyant sur ces mêmes travaux, différents auteurs ont eu recours à d'autres adsorbants imprégnés avec du nitrate d'argent. Badings et Wassink (1963) ont obtenu une bonne séparation des 
classes d'aldéhydes peu influencée par la longueur de chaîne de leurs membres en imprégnant du Kieselguhr G. Grosch et al. (1974), Harkes et Begemann (1974), Meijboom (1968) ont à la suite de Meijboom et Jurriens (1965) obtenu la séparation des classes d'aldéhydes sur gel de silice imprégné ; ce dernier utilisé dans les travaux initiaux sur les lipides n'avait pas jusque-là été rapporté comme donnant des résultats satisfaisants dans la séparation des classes d'aldéhydes.

Dans des développements récents, les travaux de Meijboom et Jongenotter (1975) sur les aldéhydes insaturés non « conventionnelles ", soulignent la remarquable aptitude du gel de silice à la séparation selon l'isomérie de position ou l'isomérie géométrique.

Si les chromatographies en couche mince d'un adsorbant imprégné de nitrate d'argent se sont trouvées bien adaptées aux recherches centrées sur l'étude des aldéhydes tels qu'en témoignent les travaux récents sur gel de silice mentionnés ci-dessus ou ceux sur gel d'alumine de Gill et al. (1974), la séparation en classes sur alumine a été supplantée par d'autres systèmes réalisant dès la première chromatographie non seulement la séparation des classes de carbonyles aliphatiques saturés mais aussi la séparation des différentes classes d'aldéhydes voire de cétones selon leur degré d'insaturation, toutes choses qui avaient exigé le recours à un adsorbant imprégné au nitrate d'argent.

Les systèmes, suivant les auteurs, se fondent sur deux principes chromatographiques différents. Les uns faisant appel aux mécanismes d'adsorption utilisent des mélanges à base d'oxyde de magnésium, plus faiblement adsorbants que l'alumine. Les autres utilisent les propriétés complexantes encore mal expliquées du carbonate de zinc.

Mettant en application le premier principe Schwartz et al. (1968) ont défini un système à base d'oxyde de magnésium et de Célite (solvant hexane-chloroforme, 95:25) ; de même Craske et Edwards (1970) ont constitué un mélange d'oxyde de magnésium et de Microcell T 38 (adsorbant à base de silicate de chaux, de très fine granulométrie : $2 \mu$ ) avec comme solvant, un mélange éther de pétrole-chloroforme, 85:35.

Parallèlement, mais s'appuyant sur le second principe Badings et Wassink (1963), ont utilisé un mélange de carbonate de zinc et d'amylopectine (solvant : éther de pétrole-benzène-pyridine, 7:1:2) tandis que Gill et al. (1974) ont mis en œuvre un système à base de carbonate de zinc et d'amidon (solvant : pyridine).

2) Séparation en homologues des différentes classes de composés carbonylés aliphatiques

C'est d'abord par chromatographie d'adsorption que les auteurs ont tenté d'obtenir la séparation des homologues d'une classe. 
Ainsi Onoe (1952) a obtenu sur gel de silice (solvant : benzène saturé d'eau) la séparation des dix premières aldéhydes saturées, du propenal, du 2-buténal et du 3,7-diméthyl-2,6-octadiénal (citral).

Dhont et De Rooy (1961) ont également proposé le gel de silice (solvant : benzène-éther de pétrole $60^{\circ}-80^{\circ}, 3: 1$ ) pour la séparation des homologues d'aliphatiques.

Sur gel d'alumine (solvant : benzène-hexane, 1:1 ou diéther oxyde) Rosmus et Deyl (1961) ont réalisé la séparation d'homologues d'aldéhydes, de cétones saturées et d'alka-1-en-3-ones.

Enfin reprenant l'étude des possibilités de la chromatographie d'adsorption pour la séparation des homologues, Badings et Wassink (1963) ont bien montré sur gel de silice que la séparation des aldéhydes saturées de $C_{2}$ à $C_{10}$ représentait la performance maximale de ce type de chromatographie.

Les recherches pour la mise au point de systèmes qui développeraient de meilleures performances ont rapidement imposé le principe d'une chromatographie de partage utilisant comme phase stationnaire polaire, un alcool de haut poids moléculaire. L'unité observée au niveau du principe chromatographique s'avérait aussi très réelle au niveau des systèmes mis en œuvre puisque malgré leur apparente diversité ces derniers peuvent être regroupés en deux catégories : ceux qui ont choisi le 2-phénoxyéthanol comme phase stationnaire et ceux qui ont retenu un polymère du P.E.G.

Urbach (1963), se réclamant des travaux de Forss et Stark (1964) qui concluaient à la supériorité du papier imprégné au 2-phénoxyéthanol pour la séparation des homologues, imaginait une chromatographie en couche mince de Kieselguhr-G imprégné de ce même alcool (solvant : éther de pétrole $100^{\circ}-120^{\circ}$ ).

Les résultats obtenus par l'auteur dans la séparation d'alkanals (séparation des homologues $C_{1}$ à $C_{14}$ ); d'alk-2-énals ( $C_{3}$ à $C_{11}$ et $C_{18}$ ), d'alka-2,4-diénals $\left(\mathrm{C}_{5}\right.$ à $\mathrm{C}_{12}, \mathrm{C}_{14}, \mathrm{C}_{16}$ et $\left.\mathrm{C}_{18}\right)$, d'alka-2-ones $\left(\mathrm{C}_{3}\right.$ à $\left.\mathrm{C}_{13}\right)$, d'alk3-en-2-ones $\left(\mathrm{C}_{6}, \mathrm{C}_{7}, \mathrm{C}_{10}\right)$ et d'alk-1-en-3-ones $\left(\mathrm{C}_{4}\right.$ à $\left.\mathrm{C}_{10}\right)$ mettaient en valeur les aptitudes de cette phase stationnaire à la séparation en homologues et engageaient d'autres auteurs, tels Schormuller (1969), à l'utiliser.

Badings et Wassink (1963) après l'étude comparative des aptitudes de divers systèmes chromatographiques de partage, portaient leur choix sur le Carbowax 400, polymère du P.E.G. avec en un premier temps comme support, le carbonate de zinc auquel ils ont substitué par la suite le Kieselguhr G, d'une mise en ouvre beaucoup plus facile. Les résultats obtenus étaient comparables à ceux atteints avec le précédent système : séparation des homologues $\mathrm{C}_{2}$ à $\mathrm{C}_{11}$ pour les alkanals (nous avons constaté que l'homologue en $\mathrm{C}_{1}$ était également séparé), $C_{4}$ à $C_{10}$ pour les alk-2-énals, $C_{8}, C_{7}, C_{8}$ et $C_{11}$ pour les alka-2,4diénals, $\mathrm{C}_{3}$ à $\mathrm{C}_{\text {n }}$ pour les 2-alkanones $\mathrm{C}_{3}, \mathrm{C}_{3}, \mathrm{C}_{7}$ et $\mathrm{C}_{10}$ pour les alk-2énones 4 . 
La simplicité de réalisation des plaques du système KieselguhrCarbowax 400, jointe à la qualité des séparations a suscité l'utilisation de ce système dans un large éventail de travaux : Boyd et al. (1965), Grosch et al. (1974), Harkes et Begemann (1974), Schormüller et al. (1969).

L'apparition du Microcell T 38 a permis à Schwartz et al. (1968) de proposer une nouvelle version du système Kieselguhr G-Carbowax 400 où ce nouvel adsorbant a remplacé le Kieselguhr G.

Du point de vue de la rapidité de confection des plaques, le système Microcell T 38 -Carbowax 400 marque un très net progrès : $10 \mathrm{mn}$ de séchage à l'air libre au lieu de $2 \mathrm{~h}$, un passage à l'étuve de $5 \mathrm{mn}$ au lieu de $30 \mathrm{mn}$. Un autre avantage est présenté par la couleur d'un blanc très pur des couches minces de Microcell T 38 qui facilite l'utilisation de révélateurs (diéthylamine par exemple) ou l'observation des couleurs caractéristiques des classes dans le cas où l'on aura confectionné les plaques avec du Carbowax 400 alcalinisé.

Malgré ses performances, le système de Badings et Wassink (1963) que ce soit sur Kieselguhr G ou dans l'adaptation seconde sur Microcell $\mathrm{T} 38$, ne peut séparer les homologues à longue chaîne, au-delà de $\mathrm{C}_{12}$ pour les alkanals, $C_{\text {, }}$ pour les 2-alkanones.

Aussi les auteurs ont-ils recherché des perfectionnements susceptibles de rendre ce système apte à la séparation des homologues supérieurs.

En utilisant une phase stationnaire alcaline (Carbowax 400 et potasse) Schwartz et al. (1968) créaient un nouveau système adapté à la séparation des termes supérieurs, le premier système continuant à être utilisé pour la séparation des homologues inférieurs. Ce nouveau système séparait bien les aldéhydes saturées et insaturées à longues chaînes mais ne séparait pas les homologues supérieurs des 2-alkanones.

Hansen et Keeney (1970) ont cherché à améliorer la séparation des homologues supérieurs dans le système Microcell T 38-Carbowax 400 sans pour autant sacrifier la séparation des termes inférieurs. Ils y sont parvenus en utilisant une phase stationnaire alcaline et en adaptant la polarité de la phase mobile à la polarité des substances à séparer : hexane saturé de Carbowax 400 ou hexane-benzène saturé de Carbowax 400 (94:6) pour les 2-alkanones, hexane-benzène saturé de Carbowax $400(85: 15)$ pour les alkanals, hexane-benzène $(65: 35)$ ou hexane-benzène saturés de Carbowax 400, pour les aldéhydes insaturés. Ainsi ont-ils pu obtenir la séparation des homologues supérieurs jusqu'au terme en $C_{16}$ pour les alkanals, $C_{11}$ pour les alk-2-énals, $\mathrm{C}_{\text {1в }}$ pour les alk-2,4-diénals.

Dans une démarche analogue, Harkes et Begemann (1974) ont amélioré le pouvoir séparateur du système initial Kieselguhr G-Carbowax 400 en substituant au polymère 400 du Carbowax, le polymère 
750. Le nouveau système appliqué à la séparation des alkanals, permettait de séparer les premiers termes.

Si ces systèmes apportaient des améliorations appréciables à la séparation des homologues supérieurs pour les classes d'aldéhydes saturées et insaturées, force était bien de constater, dans l'ensemble leur inadaptation à la séparation des 2-alkanones à longue chaîne. C'est pourquoi Hansen et Keeney (1970) ont proposé pour la séparation des 2-alkanones à longue chaîne, une chromatographie de partage à phases inversées sur support de Microcell T 38 avec comme phase stationnaire apolaire, une huile minérale (Nujol) et comme solvant polaire un mélange acétonitrile-eau (85-15). Les problèmes de solubilité liés au poids moléculaire élevé des substances ne leur ont pas permis d'obtenir des séparations complètes mais par cette technique ils ont pu néanmoins au cours de chaque essai obtenir une séparation satisfaisante pour un certain nombre d'homologues supérieurs des 2-alkanones.

Au cours de ces chromatographies l'identification des homologues pourra souvent être obtenue, conjointement à leur séparation par comparaison avec la séparation d'un mélange témoin déposé sur la même plaque.

\section{III. - Séparation des composés carbonylés aromatiques}

Les composés carbonylés aromatiques constituent avec les composés dicarbonylés sous forme d'osazones, l'essentiel de la fraction 5 isolée au cours de la séparation en groupes de classes (fig. 2). D’autres composés carbonylés peuvent accessoirement y être représentés ; mais comme ils sont toujours en nombre limité, leur séparation pourra souvent être réalisée sans grande difficulté conjointement à celle des aromatiques. Leur identification généralement obtenue par utilisation d'un mélange témoin pourra se trouver facilitée dans certains cas par le développement de couleurs caractéristiques pour le dérivé mono du diacétyle ou pour le méthanal par exemple. Enfin il doit être souligné que parmi ces composés, les composés aliphatiques - méthanal et les premiers termes des alk-2-énals - pourront, si le protocole ci-dessus apparaît difficilement réalisable, être plus aisément séparés en les conservant dans la fraction aliphatique (fraction 1, système I) justiciable des méthodes de séparation préconisées plus haut (séparation en classes puis en homologues des composés aliphatiques).

La grande diversité chimique des composés aromatiques rencontrés - ce ne sont pas en général les homologues d'une même classe confère à chacun d'entre eux un comportement chromatographique original même si, comme a pu le montrer Ruffini (1965), des corrélations peuvent être établies entre la constitution chimique et le comportement chromatographique dans le cas de certaines aldéhydes et cétones aromatiques hydroxylées. 
La séparation et l'identification de ces composés ne pourront donc être entreprises qu'à la seule échelle individuelle. Du point de vue de la séparation le nombre élevé des composés susceptibles d'être présents dans un mélange ne permettra pas d'obtenir des résultats satisfaisants pour tous les composés avec un seul système chromatographique. Certains composés auront un comportement chromatographique trop voisin pour être séparés s'ils sont présents simultanément dans le même mélange.

Pour obvier cette difficulté Dhont et Dijkman (1967) ont imaginé un ensemble de 7 systèmes (tab. 1) capables de susciter chez

\section{TABLEAU 1}

Systèmes chromatographiques utilisés pour la séparation des composés carbonylés aromatiques d'après Dhont et Dijkman (1967)

\begin{tabular}{|c|c|c|c|}
\hline Système & Adsorbant & Solvant & $\begin{array}{c}\text { Nombre } \\
\text { de } \\
\text { dévelop- } \\
\text { pements }\end{array}$ \\
\hline III & Cellulose acétylée & $\begin{array}{l}\text { Ether de pétrole-méthanol-acétate d'éthyle } \\
9,5: 1,5: 1,0\end{array}$ & 2 \\
\hline IV & Cellulose acétylée & $\begin{array}{l}\text { Ether de pétrole-oxyde dibutylique-acétate } \\
\text { d'éthyle-méthanol, 65:10:15:10 }\end{array}$ & 1 \\
\hline V & Cellulose acétylée & $\begin{array}{l}\text { Ether de pétrole-oxyde dibutylique-acétate } \\
\text { d'éthyle-méthanol, 85:5:5:5 }\end{array}$ & 1 \\
\hline VI & Gel de silice G & $\begin{array}{l}\text { Ether de pétrole-benzène, } \\
1: 1\end{array}$ & 5 \\
\hline VII & Gel de silice $G$ & $\begin{array}{l}\text { Ether de pétrole-benzène- oxyde } \\
\text { dibutylique }\end{array}$ & 1 \\
\hline VIII & Carbonate de zinc & $\begin{array}{l}\text { Ether de pétrole-pyridine, } \\
8: 1\end{array}$ & 1 \\
\hline IX & Anasil B & $\begin{array}{l}\text { Ether de pétrole-benzène-pyridine, } \\
8: 1: 0,25\end{array}$ & 2 \\
\hline
\end{tabular}

un même composé des comportements suffisamment différents pour que d'un système à l'autre les composés se présentent dans un ordre relatif différent. Ainsi tels composés occupant des localisations contiguës non distinctes, se retrouveront dispersés dans d'autres systèmes. 
Ces systèmes qui utilisent tous la p-tolualdéhyde comme composé marqueur permettent d'attribuer à chaque composé un $\mathrm{R}_{\mathrm{s}}$ : $\mathrm{R}_{\mathrm{F}}$ de la substance

$\mathrm{R}_{\mathrm{F}}$ du marqueur correspondant à la substance et au système.

L'ensemble des différentes valeurs du $R_{s}$ sera caractéristique du composé et permettra son identification.

Dans ces divers systèmes les dérivés bis des composés dicarbonylés plus polaires que les DNPHones des composés aromatiques migreront peu ou resteront sur la ligne de dépôt et seront ainsi isolés. Par ailleurs les couleurs rouge ou violet des osazones faciliteront leur repérage.

\section{IV. - Séparation des composés dicarbonylés}

Compte tenu du nombre limité d'homologues, constituant chaque classe, le protocole de séparation (fig. 1) pourra être amputé du stade B de séparation en sous-groupes de classes.

\section{1) Séparation en classes}

La séparation en classes qui, comme pour les composés aliphatiques, s'effectue par chromatographie d'adsorption, sur un mélange à base d'oxyde de magnésium, doit pour tenir compte du caractère très polaire des composés dicarbonylés adapter la polarité du solvant par addition de méthanol et parfois limiter les propriétés d'adsorption de l'oxyde de magnésium.

Cobb (1964) a obtenu la séparation des $\alpha$-dicétones, $\alpha$-cétoalkanals et du glyoxal avec deux systèmes à base de Sea Sorb 43 (oxyde de magnésium activé) : système a) - Sea Sorb 43 - Silicagel G (1:1), système b) - Sea Sorb 43 - célite - sulfate de calcium $(10: 8,5: 1,5)$ avec comme solvant soit un mélange de benzène saturé d'éthanolamine et de méthanol (92:8), soit un mélange de chloroforme, de tétrahydrofurane et de méthanol $(15: 4: 1)$. Les premiers termes des $\alpha$-dicétones et des a-cétoalkanals - respectivement le diacétyle et le méthylglyoxal - sont dès cette première étape de fractionnement en classes, séparés de leurs homologues et identifiables.

Schwartz et al. (1964) ont proposé un système à base de Sea sorb 43 partiellement désactivé : Sea Sorb 43 - Célite 545 (15:6). La difficulté à obtenir une désactivation de même niveau pour chaque lot de Sea Sorb les a conduit à adopter comme solvant un mélange acétone-benzène-méthanol de polarité variable $(75: 23: 2,75: 21,5: 3,5$, $75: 20: 5$ ou 75:15:10), la variante la mieux adaptée au pouvoir adsorbant du lot de Sea Sorb étant choisie après essai. Ce système permet de réaliser un fractionnement identique à celui obtenu avec le système précédent.

Schwartz et al. (1968) s'affranchissent de l'opération délicate de désactivation partielle du Sea Sorb 43 en créant un système à base d'oxyde de magnésium non activé et de Célite (solvant chloroforme - 
méthanol, 95:5) qui donne des résultats en tous points comparables aux précédents.

\section{2) Séparation des homologues}

C'est par chromatographie de partage à phases inversées que Cobb et al. (1965) ont obtenu la séparation des homologues des deux classes d' $\alpha$-dicarbonylés ( $\alpha$-dicétones et $\alpha$-cétoalkanals). Ils ont imprégné un support de gel de silice $\mathrm{G}$ avec comme phase stationnaire apolaire une huile minérale (Shell Ondina 27 ou Nujol) et ont choisi comme solvant polaire un mélange dioxane-eau (6:4). Ce système présente une meilleure aptitude à la séparation des $\alpha$-cétoalkanals qu'à la séparation des $\alpha$-dicétones puisqu'il sépare les premières jusqu'à l'homologue en $C_{10}$ tandis qu'il ne sépare les dernières que jusqu'à l'homologue en $\mathrm{C}_{8}$.

La mise en cuvre par Schwartz et al. (1968) du Carbowax 400 sur support de Microcell T 38, solvant : benzène-hexane (3:2) pour la séparation des $\alpha$-cétoaldéhydes (1:3), pour la séparation d' $\alpha$-dicétones, ne marque pas un progrès du point de vue de la qualité des séparations, mais elle permet de réaliser un gain de temps très important.

\section{CONCLUSION}

La succession des systèmes chromatographiques proposés dans cet article démontre la possibilité d'obtenir la séparation d'un mélange complexe de composés carbonylés par la seule technique de chromatographie en couches minces.

De plus elle établit un schéma de séparation qui, dicté par la constitution chimique des composés, acquiert une portée méthodologique générale autorisant, pour la réalisation de certaines des étapes, la substitution d'une autre technique chromatographique à la chromatographie en couches minces. C'est ainsi par exemple que la chromatographie sur colonne en phase liquide, appliquée à la séparation en sous-groupes de classes (Etape B, fig. 1) et en classes (Etape C, fig. 1) permettra parce qu'elle pourra séparer des quantités d'échantillons beaucoup plus importantes que par couches minces, d'éviter la répétition des fractionnements avant le stade final de séparation en homologues.

\section{Ré s u m é}

La séparation des 2,4-DNPHones des composés carbonylés peut être obtenue par un enchaînement de chromatographies en couche mince qui dans le cas le plus complexe, celui des composés aliphatiques, comprend quatre étapes : 
(A) Fractionnement en groupes de classes. Le groupe des aliphatiques est séparé des aromatiques et des dicarbonylés réunis.

(B) Fractionnement des aliphatiques en trois sous-groupes à chaînes courtes, moyennes et longues.

(C) Fractionnement en classes.

(D) Séparation des classes en leurs homologues.

La séparation des aromatiques est obtenue directement sans fractionnement préalable en sous-groupes de classes et en classes. Les dicarbonylés, isolés au cours de cette séparation sont séparés en mettant en œuvre les étapes C et D (étape B supprimée).

\section{S u m m a r y}

Separation of the 2,4-DNPHones of carbonyls can be performed by a serie of TLC ; for the more complex group i.e., aliphatics, four steps are needed.

Step A. Separating in group of class by which aliphatics are made free of aromatics and dicarbonyls.

Step B. Separating aliphatics in three groups according to chain length (short, medium, long chains).

Step C. Class separation.

Step B. Separating individual compounds within a class.

Aromatics obtained by step A are directly separated according to step D. Dicarbonyls thus isolated are fractionated by step $C+D$ (omitting B).

Reçu pour publication en juillet 1975.

\section{Références}

Anderson (D. F.) (1966). - Flavor chemistry of blue cheese. Diss. Abstr., 26 (6), 6636-6637.

ANDERSON(D.F.) and Day (E.A.) (1966). - Quantitation, evaluation and effect of certain microorganisms on flavor components of blue cheese. J. Agr. Food Chem., 14, 141-145.

BADINGS (H. T.) and WASSINK (J.G.) (1963). - Separation and identification of aliphatic aldehydes and ketones by thin-layer chromatography of the 2,4dinitrophenylhydrazones. Neth. Milk Dairy J., 17, 132-149.

Barrett (C. B.), Dallas (M. S. J.) and Padley (F. B.) (1962). - The separation of glycerides by thin-layer chromatography on silica impregnated with silver nitrate. Chem. Ind., 1050-1051.

Boyd (E. N.), Keeney (P. G.) and Patton (S.) (1965). - The measurement of monocarbonyl classes in cocoa beans and chocolate liquor with special reference to flavor. J. Food Sci., 30 (5), 854-859. 
CoвB (W. Y.) (1964). - Separation of 2,4-dinitrophenylhydrazones of vicinal dicarbonyls into classes by thin-layer chromatography. J. Chromat., 14, 512-513.

CoBB (W. Y), LiBBEY (L. M.) and DAY (E. A.) (1965). - Thin-layer chromatography of 2,4-dinitrophenylosazone homologs of vicinal dicarbonyls. J. Chromat., 17, 606-607.

CRASKe (J. D.) and EdWARDS (R. A.) (1970). - A two dimensional thin-layer chromatographic technique for the resolution of monocarbonyl dinitrophenylhydrazones. J. Chromat., 51, 237-241.

DARTEY (C. K) and Kinsella (J. E.) (1971). - Rate of formation of methyl ketones during blue cheese ripening. J. Agr. Food Chem., 19 (4), 771-774.

DAY (E. A.) (1965), - Some properties of carbonyl compounds encountered in the flavor isolates of dairy products. A review. Food Technol., 19 (2), 1585-1590.

Dhont (J.H) and DiJKman (G.J.C.) (1967). - The quantitative analysis of complexe carbonyl mixtures by thin-layer chromatography. Analyst, 92, 431-435.

DHont (J.H,) and DE RooY (C.) (1961). - Chromatographic behaviour of 2,4dinitrophenylhydrazones on chromatoplates. The Analyst, 86, 74-76.

Forss (D. A.), Dunstone (E. A.) and Stark (W.) (1960). - Fractionation of complex mixtures of 2,4-dinitrophenylhydrazones. Aust. J. Chem., 13 (4), 584-586.

Forss (D. A.), Pont (E. G.) and STARK (W.) (1955). - The volatile compounds associated with oxidized flavour in skim milk. J. Dairy Res., 22, 91-102.

Forss (D. A.) and Stark (W.) (1964). - Paper chromatography of the 2,4-dinitrophenylhydrazones of alk-1-en-3-ones. Anal. Chem., 36, 941-942.

Gill Mc (A. S.), Hardy (R.), Burt (J. R.) and Gunstone (F. D.) (1974), - Hept-cis4-enal and its contribution to the off-flavour in cold stored cod. J. Sci. Fd Agric., 25, 1477-1489.

Grosch (W.), Laskawy (G.) and Fischer (K. H.) (1974). - Oxidation of linolenic acid in the presence of haemoglobin, lipoxygenase or by singlet oxygen. Identification of the volatile carbonyl compounds. Lebensmitt. Wiss. u. Technol., 7 (6), 335-338.

HARKes (P.D.) and BegemanN (W. J.) (1974). - Identification of some previously unknown aldehydes in cooked chicken. Jaocs, 51 (8), 356-359.

Haverkamp Begemann (P.) and DE Jong (K.) (1959). - Analytical applications of a celite-dinitrophenylhydrazine column. Rec. Trav. Chim., 78, 275-283.

HUELIN (F. E.) (1952). - Volatile products of apples. III. Identification of aldehydes and ketones. Aust. J. Sci. Res., Series B, 5, 328-334.

Kallio (H.), Linko (R. R.) and Kaitaranta (J.) (1972). - Gas-liquid chromatographic analysis of 2,4-dinitrophenylhydrazones of carbonyl compounds. J. Chromat., 65, 355-360.

Meisвoom (P. W.) (1968). - Die Trennung und Identifizierung der 2,4-Dinitrophenylhydrazone ungesättigter Aldehyde und Methylketone mittels Dünnschicht-Chromatographie. Fette Seifen Anst., 70 (7), 477-481.

Meijooom (P. W.) und JongenotTer (G. A.) (1975). - Das chromatographische Verhalten der 2,4-Dinitrophenylhydrazone aliphatischer ungesättigter Aldehyde auf mit Silbernitrat imprägnierten Kieselgel-Dünnschichten. Fette Seifen Anst., 77, 135-138.

MeiJboom (P. W.) and Jurriens (G.) (1965). - Separation of positional and geometrical isomers of monoenoic aldehydes via the dinitrophenylhydrazones. J. Chromat., 18, 424-426.

Morgan (M. E.) and ANDERson (E. O.) (1956). - The neutral carbonyl compounds in blue-mold type cheese. J. Dairy Sci., 39, 253-260. 
ONOE (K.) (1952). - The separation and identification of aliphatic aldehyde 2,4-dinitrophenylhydrazones by the chromatostrip method. J. Chem. Soc., Japan, Pure Chem. Sect., 73, 337-339. In : C.A., 47 (1953), 3757 d.

PAPA (L. J.) and TURNER (L. P.) (1972). - Chromatographic determination of carbonyl compounds as their 2,4-dinitrophenylhydrazones. I. Gas chromatography. J. Chromat. Sci., 10, 744-747.

Patton (S.) (1950). - The methyl ketones of blue cheese and their relation to its flavor. J. Dairy Sci., 33 (9), 680-684.

Reich (H.) and Hefle (L.) (1956). - The mono-2,4-dinitrophenylhydrazones of diacetyl and glyoxal. J. Org. Chem., 21, 708.

Rosmus (J.) and DeYL (Z) (1961). - Two improved methods for the separation of 2,4-dinitrophenylhydrazones of carbonyl compounds. J. Chromat., 6, 187-190.

RUFFINI (G.) (1965). - Thin-layer chromatography of 2,4-dinitrophenylhydrazones of aromatic aldehydes and ketones. J. Chromat., 17, 483-487.

Sato (M.), Honda (T.), Yamada (Y.), Takada (A.) and Kawanami (T.) (1966). - A study on free fatty acids, volatile carbonyl compounds and tyrosine in blue cheese. XVII ${ }^{\mathrm{e}}$ Congr. Int. Lait., $D$, 539-544.

Schormüller (J.), Walther (M.) und Wachs (W.) (1969). - Veränderungen von Milchproteinen durch Einwirkung von Carbonylverbindungen III Neutrale Monocarbonylverbindungen in gelagerter, sprühgetrockneter Vollmilch. $Z$. Lebens. Unter., 139 (5), 273-282.

Schwartz (D. P.), KeEney (M.) and Parks (O. W.) (1964). - Thin-layer chromatography of carbonyl compounds. Separation of dicarbonyl bis (2,4-dinitrophenylhydrazones) into classes. Microchem. J., 8, 176-179.

SchwartZ (D. P.) and PARKS (O.W.) (1963). - Quantitative analysis of methyl ketones in blue cheese fat. J. Dairy Sci., 46 (9), 989-990.

Schwartz (D. P.), Shamey (J.), Brewington (C. R.) and Parks (O. W.) (1968). Methods for the isolation and characterization of constituents of natural products. X. New and improved methods for the analysis of carbonyl 2,4dinitrophenylhydrazones and 2,4-dinitrophenylosazones. Microchem. J., 13 (3), 407-417.

Soukup (R. J.), Scarpellino (R. J.) and Danielczik (E.) (1964). - Gas chromatographic separation of 2,4-dinitrophenylhydrazone derivatives of carbonyl compounds. Anal. Chem., 36, 2255-2256.

URBACH (G.) (1963). - Thin-layer chromatography of aliphatic 2,4-dinitrophenylhydrazones. J. Chromat., 12, 196-207.

DE VRIES (B.) (1962). - Quantitative separations of lipids materials by column chromatography on silica impregnated with silver nitrate. Chem. Ind., 1049-1050. 